\title{
The pivotal role of partnership between media and medical institutions: A healthy start during coronavirus disease-19 era
}

\author{
Swayam Pragyan Parida ${ }^{1}$, Vikas Bhatia ${ }^{2}$, Sonali Sinha ${ }^{3}$, Prajna Parimita Giri ${ }^{4}$, Binod Kumar Behera ${ }^{1}$ \\ From ${ }^{1}$ Associate Professor, ${ }^{2} \mathrm{Head},{ }^{3}$ Senior Consultant, ${ }^{4}$ Assistant Professor, Department of Community Medicine and Family Medicine, All India \\ Institute of Medical Sciences, Bhubaneswar, Odisha, India
}

T he role of media is crucial in shaping and influencing people's attitudes and behavior. It has a responsibility to report and educate the public about accurate health information [1]. In this age of digital revolution, the health professionals must engage with media in the most effective manner so that the communities at large get the right information. Media when used cautiously provides obvious advantages such as clinical education and health promotion. However, when used unwisely, social media has its disadvantages too such as violation of patients' confidentiality and privacy, flaring up unnecessary issues, and misinformation, which may lead to formidable consequences [2].

Social media channels may also be used as part of the training process of health-care professionals to make adult learning more enjoyable and interactive. Social media gives participants the power to provide presenters with immediate feedback on training sessions. Microblogs provide the most dynamic and concise form of information exchange through social media by allowing users to post a large number of brief messages or updates over a short period. Twitter and other social media platforms are also being utilized by health-care professionals to provide up-to-date information during procedures to fellow doctors or medical students [3,4].

Media has been playing a key role in the coronavirus disease (COVID-19) response. Health-care professionals and media have been working relentlessly with the government machinery in containing the COVID-19 outbreak, promoting good behaviors on health and hygiene, breaking myths, and misconceptions including stigmatization, thereby potentially, saving lives to the optimal. During the ongoing pandemic, the government has been using media, to deliver real-time updates on a number of positive cases, hospital capacity, medical status of patients, and other precautionary measures to be followed by the citizens. Telemedicine/online consultations are getting better day by day [5] In addition, engaging with community radio predominantly in media dark areas has been giving an added advantage to reach out to the most vulnerable sections of the society.

\section{Access this article online}

Received - 13 July 2020

Initial Review - 25 August 2020

Accepted - 06 October 2020

DOI: 10.32677/IJCH.2020.v07.i10.010
To build confidence and conviction and to minimize false perceptions developed from fake news, it is highly pertinent to be able to develop a relationship of trust, a partnership between medical institutions and media for sharing true information and knowledge to the people. Each medical college/health institution may appoint a nodal person or public relations officer for engaging with media. This may prove to be beneficial in actual reporting and contain fake/biased reports. Toward achieving this objective, AIIMS Bhubaneswar in Odisha from eastern India has been organizing media sensitization cum engagement workshops. A total of three such workshops have been completed (one being a webinar) showing an overwhelming response from the media houses and also from the medical fraternity, throwing open a roadmap on building trust to bridge gaps. Therefore, it is important to create positive networking with medical/public health professionals, leading to a win-win situation not only for media and medical fraternity but also for the general masses at large.

\section{REFERENCES}

1. Hakim KM, Akhtar NB, Hakeem RM. Impacts of media on society: A sociological perspective. Int J Humanit Soc Sci Invent 2014;3:56-64.

2. Bernhardt M, Alber J, Gold RS. A social media primer for professionals: Digital dos and don'ts. Health Promot Pract 2014;15:168-72.

3. Grajales FJ, Sheps S, Ho K, Novak-Lauscher H, Eysenbach G. Social media: A review and tutorial of applications in medicine and health care. J Med Internet Res 2014;16:e13.

4. Chauhan B, George R, Coffin J. Social media and you: What every physician needs to know. J Med Pract Manage 2012;28:206-9.

5. WHO Health Alert Brings COVID-19 Facts to Billions via WhatsApp. Available from: https:/www.who.int/news-room/feature-stories/detail/whohealth-alert-brings-covid-19-facts-to-billions-via-whatsapp. [Last accessed on 2020 Sep 22].

Funding: None; Conflicts of Interest: None Stated.

How to cite this article: Parida SP, Bhatia V, Sinha S, Giri PP, Behera BK. The pivotal role of partnership between media and medical institutions: A healthy start during coronavirus disease-19 era. Indian J Child Health. 2020; 7(10):430.

Correspondence to: Sonali Sinha, Resource Centre for Advancement on Public Health Nutrition, All India Institute of Medical Sciences, Bhubaneswar Odisha, India. E-mail: ssonali.sss@gmail.com

(C) 2020 Creative Commons Attribution-NonCommercial 4.0 International License (CC BY-NC-ND 4.0). 